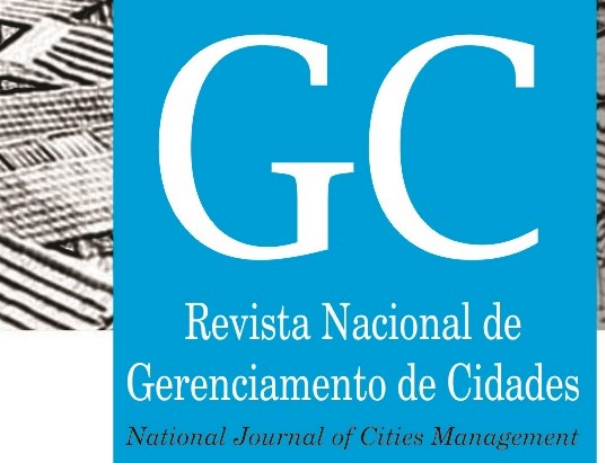

\title{
Procedimento para Construção da Equação de Chuva a partir das máximas precipitações diárias obtidas de séries anuais: Atualização da Equação de Chuva da cidade de São Carlos (SP)
}

Procedure for Construction of Rain Equation from the Maximum Daily Rainfall obtained from anual series: Rain Equation Update of the city of São Carlos (SP)

Procedimiento para la Construcción de la Ecuación de la lluvia em vista de las máximas precipitaciones diárias obtenidas de serie anual: Actualización de la ecuación de la Lluvia de la ciudad de San Carlos (SP)

Lucas Narciso de Britto Engenheiro Civil, UFSCar, Brasil. Inbritto@gmail.com

Erich Kellner Professor Doutor, UFSCar, Brasil. erich.kellner@ufscar.br 


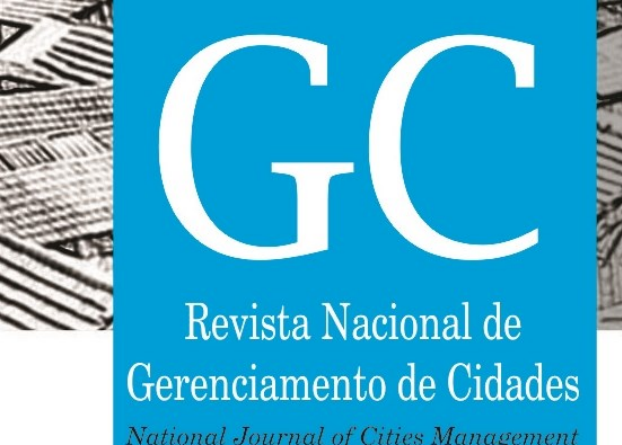

\section{1 - INTRODUÇÃO}

A Hidrologia é a ciência responsável por tratar da água no planeta Terra, além da sua circulação e relação com o meio ambiente e as formas vivas. Apresentada como uma ciência interdisciplinar que tem evoluído significativamente em face aos problemas resultantes da ocupação das bacias e do aumento significativo na utilização de água, a Hidrologia passou de uma ciência preponderantemente descritiva e qualitativa para uma área do conhecimento que se utiliza de métodos matemáticos e estatísticos como forma de analisar as informações existentes.

O ser humano convive desde a sua origem com as condições naturais do planeta, sendo essencial para a sobrevivência deste o entendimento dos processos envolvidos no meio físico natural. Para o caso das precipitações, por exemplo, são utilizadas observações realizadas no passado para tentar entender a sazonalidade e a intensidade das suas ocorrências.

Ao ocupar determinada região das bacias hidrográficas, ocorre de forma simultânea o impacto sobre o meio ambiente e o impacto do meio ambiente sobre o homem, através das enchentes. Surge, então, a necessidade de um estudo aprofundado sobre a relação entre as precipitações e a ocupação da bacia pelo ser humano, dando origem a uma das principais áreas da Hidrologia Aplicada: a Drenagem Urbana (TUCCl, 2002).

Um sistema de Drenagem Urbana faz parte dos melhoramentos públicos efetuados na infraestrutura, pavimentação de ruas, guias e passeios, dentre outros. É ideal para a comunidade que a área urbana seja concebida de forma integrada, ou seja, todos os melhoramentos públicos sejam elaborados de forma conjunta. Caso o sistema de drenagem não seja considerado desde o início da formulação do planejamento, por exemplo, este pode revelar-se no futuro como um sistema de alto custo e ineficiente.

O sistema de drenagem urbana possui uma particularidade: o escoamento das águas pluviais sempre ocorrerá independente da existência ou não deste sistema. A qualidade do sistema projetado é que determinará os benefícios ou prejuízos à população (CETESB, 1978).

A forma de utilização dos dados de precipitações pluviais para finalidades hidrológicas é feita a partir do estabelecimento de uma relação analítica entre a intensidade, a duração e a frequência das precipitações. Essa relação, denominada equação de chuva, permite uma maior eficácia no projeto de sistemas de drenagem urbana (WILKEN, 1978).

A equação de chuva é obtida a partir de dados de precipitações pluviais passados, como por exemplo, séries históricas anuais. Porém, com o passar dos anos existem indícios de que os dados pluviais podem sofrer alterações por motivos diversos, tornando a equação de chuva distante da realidade e implicando em dimensionamentos imprecisos das obras hidráulicas públicas, conforme apontado por Zuffo (2004), em uma análise feita para a cidade de Campinas (SP).

A cidade de São Carlos (SP) tem sua equação de chuva proposta por Barbassa (1991), que contempla 27 anos de dados de precipitações e ainda é utilizada em projetos de drenagem urbana. Por conta disso, surge a necessidade de atualizar a equação I-D-F incorporando os últimos 25 anos de dados referentes às máximas precipitações diárias. 


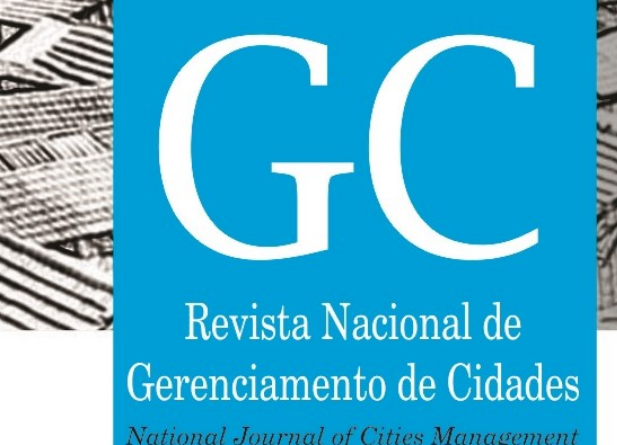

Cavalcanti, Silva e Reis (2015) usaram os dados de precipitação da Estação Convencional do campus da Universidade Federal de São Carlos como base para análise de consistência de outras estações de medição, sendo ela considerada confiável para análise de consistência dos postos de monitoramento instalados mais recentemente na malha urbana da cidade.

Pela inexistência de Estações Pluviométricas na mesma região hidrológica e com período histórico tão longo quanto o da Estação Convencional localizada na UFSCar não foram aplicados métodos de análise de consistência de dados para a estação em questão, conforme sugerido por ANA (2012). Todavia, a confiabilidade dos dados da Estação Convencional utilizada como fonte deste trabalho não foi afetada pela falta de análises de consistência.

Os dados pluviométricos da Estação Convencional №83726 do INMET foram obtidos do endereço eletrônico www.inmet.gov.br abrangendo o período de 01/01/1961 a 31/12/2015.

\section{2 - Preparação dos dados a serem analisados}

Da análise da série histórica de precipitação registrada pela Estação Convencional no83726 do INMET foram considerados os anos que apresentaram menos de 10 dias de falhas no ano, ou que não tiveram falhas no período chuvoso.

O conjunto de dados de precipitação referente a um determinado ano que não se enquadrou nesses critérios foi desconsiderado para a análise das máximas precipitações.

\section{3 - Análise estatística dos dados pluviométricos}

\subsection{1 - Verificação de ocorrência de Outliers}

Os dados pluviométricos foram submetidos a análises estatísticas a fim de se obter médias e desvios-padrão anuais, bem como a distribuição de frequência.

A partir do conjunto de dados relativos às máximas precipitações foi avaliada a ocorrência de outlier conforme descrito por Pinheiro (2013).

- $\quad$ Os valores anuais de precipitação máxima foram colocados em ordem crescente;

- Identificou-se a mediana;

- Identificou-se o menor e o maior número da série;

- Identificou-se a mediana entre o menor número da série e a mediana geral $\left(Q_{1}\right)$;

- Identificou-se a mediana entre o maior número da série e a mediana geral $\left(Q_{3}\right)_{i}$

- $\quad$ O cálculo do interquartil (IQR) ocorreu conforme a Equação (1):

- $\quad$ O limite de outlier $\left(\mathrm{L}_{0}\right)$ foi obtido pela Equação (2):

$$
I Q R=Q_{3}-Q_{1}
$$

$$
L_{0}=I Q R \times 1,5
$$

Foram considerados outliers os números da série abaixo de $\left(Q_{1}-L_{0}\right)$ ou acima de $\left(Q_{3}+L_{0}\right)$.

Da série histórica, foram desconsiderados os anos cujas máximas precipitações estavam fora deste intervalo, a fim de se garantir um bom ajuste ao empregar a formatação de Gumbel, conforme proposto por Pinheiro (2013).

\subsection{2 - Verificação da aderência da Série Pluviométrica à curva de Gumbel}

Após a verificação da ocorrência de outliers e da determinação da série pluviométrica a ser considerada, os dados foram dispostos em ordem decrescente e foram calculadas a probabilidade de ocorrência e o período de retorno $\left(T_{R}\right)$ equivalente, sendo construído o gráfico da máxima precipitação em função do período de retorno. 


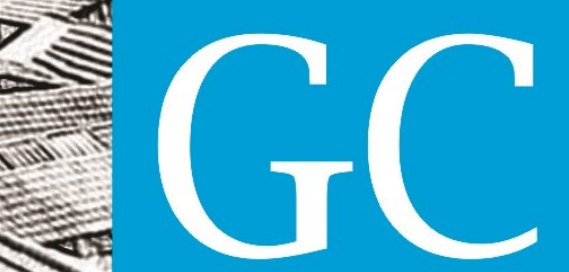

Revista Nacional de

A partir da média $(\bar{P})$ e desvio padrão $\left(\sigma_{x}\right)$ da série pluviométrica considerada foram calculados os valores de K de Gumbel corrigidos conforme Equação (3) proposta por Subramanya (2013), para determinação dos períodos de retorno.

$$
K=\frac{-\left\{\ln \left[\ln \left(\frac{T_{R}}{T_{R}-1}\right)\right]\right\}-y_{n}}{\sigma_{n}}
$$

Sendo $y_{n}$ a média reduzida e $\sigma_{n}$ é o desvio padrão reduzido, ambos relativos ao tamanho da amostra e obtidos das Tabelas 1 e 2 , respectivamente.

Tabela 1: Valores da média reduzida $\mathrm{y}_{\mathrm{n}}$ para o método de Gumbel em função do tamanho da amostra $\mathrm{N}$.

\begin{tabular}{ccccccccccc}
\hline $\begin{array}{c}\text { Tamanho } \\
\text { da amostra } \\
\mathbf{N}\end{array}$ & $\mathbf{0}$ & $\mathbf{1}$ & $\mathbf{2}$ & $\mathbf{3}$ & $\mathbf{4}$ & $\mathbf{5}$ & $\mathbf{6}$ & $\mathbf{7}$ & $\mathbf{8}$ & $\mathbf{9}$ \\
\hline 10 & 0,4952 & 0,4996 & 0,5035 & 0,5070 & 0,5100 & 0,5128 & 0,5157 & 0,5181 & 0,5202 & 0,5220 \\
20 & 0,5236 & 0,5252 & 0,5268 & 0,5283 & 0,5296 & 0,5309 & 0,5320 & 0,5332 & 0,5343 & 0,5353 \\
30 & 0,5362 & 0,5371 & 0,5380 & 0,5388 & 0,5396 & 0,5402 & 0,5410 & 0,5418 & 0,5424 & 0,5430 \\
40 & 0,5436 & 0,5442 & 0,5448 & 0,5453 & 0,5458 & 0,5463 & 0,5468 & 0,5473 & 0,5477 & 0,5481 \\
50 & 0,5485 & 0,5489 & 0,5493 & 0,5497 & 0,5501 & 0,5504 & 0,5508 & 0,5511 & 0,5515 & 0,5518 \\
60 & 0,5521 & 0,5524 & 0,5527 & 0,5530 & 0,5533 & 0,5535 & 0,5538 & 0,5540 & 0,5543 & 0,5545 \\
70 & 0,5548 & 0,5550 & 0,5552 & 0,5555 & 0,5557 & 0,5559 & 0,5561 & 0,5563 & 0,5565 & 0,5567 \\
80 & 0,5569 & 0,5570 & 0,5572 & 0,5574 & 0,5576 & 0,5578 & 0,5580 & 0,5581 & 0,5583 & 0,5585 \\
90 & 0,5586 & 0,5587 & 0,5589 & 0,5591 & 0,5592 & 0,5593 & 0,5595 & 0,5596 & 0,5598 & 0,5599 \\
$\geq 100$ & 0,5600 & - & - & - & - & - & - & - & - & - \\
\hline
\end{tabular}

Fonte: Subramanya (2013)

Tabela 2: Valores do desvio padrão reduzido $\sigma_{n}$ para o método de Gumbel em função do tamanho da amostra $N$.

\begin{tabular}{cccccccccccc}
\hline $\begin{array}{c}\text { Tamanho } \\
\text { da amostra } \\
\mathbf{N}\end{array}$ & $\mathbf{0}$ & $\mathbf{1}$ & $\mathbf{2}$ & $\mathbf{3}$ & $\mathbf{4}$ & $\mathbf{5}$ & $\mathbf{6}$ & $\mathbf{7}$ & $\mathbf{8}$ & $\mathbf{9}$ \\
\hline 10 & 0,9496 & 0,9676 & 0,9833 & 0,9971 & 1,0095 & 1,0206 & 1,0316 & 1,0411 & 1,0493 & 1,0565 \\
20 & 1,0628 & 1,0696 & 1,0754 & 1,0811 & 1,0864 & 1,0915 & 1,0961 & 1,1004 & 1,1047 & 1,1086 \\
30 & 1,1124 & 1,1159 & 1,1193 & 1,1226 & 1,1255 & 1,1285 & 1,1313 & 1,1339 & 1,1363 & 1,1388 \\
40 & 1,1413 & 1,1436 & 1,1458 & 1,1480 & 1,1499 & 1,1519 & 1,1538 & 1,1557 & 1,1574 & 1,1590 \\
50 & 1,1607 & 1,1623 & 1,1638 & 1,1658 & 1,1667 & 1,1681 & 1,1696 & 1,1708 & 1,1721 & 1,1734 \\
60 & 1,1747 & 1,1759 & 1,1770 & 1,1782 & 1,1793 & 1,1803 & 1,1814 & 1,1824 & 1,1834 & 1,1844 \\
70 & 1,1854 & 1,1863 & 1,1873 & 1,1881 & 1,1890 & 1,1898 & 1,1906 & 1,1915 & 1,1923 & 1,1930 \\
80 & 1,1938 & 1,1945 & 1,1953 & 1,1959 & 1,1967 & 1,1973 & 1,1980 & 1,1987 & 1,1994 & 1,2001 \\
90 & 1,2007 & 1,2013 & 1,2020 & 1,2026 & 1,2032 & 1,2038 & 1,2044 & 1,2049 & 1,2055 & 1,2060 \\
$\geq 100$ & 1,2065 & - & - & - & - & - & - & - & - & - \\
\hline \multicolumn{7}{r}{ Fonte: Subramanya (2013) }
\end{tabular}

Com os valores de K de Gumbel corrigido, obtiveram-se as precipitações máximas de Gumbel conforme Equação (4).

$$
P_{T}=\bar{P}+K \times \sigma_{x}
$$




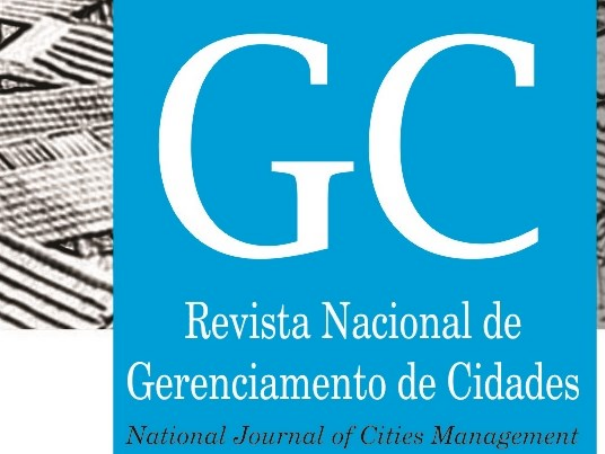

\subsection{1 - Determinação da constante "c"}

De acordo com Wilken (1978), para o cálculo da constante c aplica-se o método de Fair-Greyer, que pressupõe a ocorrência de um evento a cada N/5 anos. Dessa maneira, o período de retorno foi fixado para a determinação da constante $c$, de acordo com a Equação (6).

$$
T_{R}=\frac{N}{5}
$$

Sendo $T_{R}$ o período de retorno (anos) fixado a partir do número $N$ de observações utilizado.

A definição do parâmetro "A" é obtida pela aplicação da Equação (7).

$$
A=a \times T_{R}^{b}
$$

Substituindo a Equação (7) em (5), obteve-se a Equação (8).

$$
I=\frac{A}{(t+c)^{d}}
$$

Em seguida foi plotado um gráfico bilogaritmo das durações de chuva em função das intensidades de chuva. Nesse gráfico, foram denominados os pontos $\left(I_{1}, t_{1}\right)$ e $\left(I_{2}, t_{2}\right)$ como sendo os pontos inicial e final da curva, respectivamente. Também foi determinado a partir desta curva o ponto $\left(I_{3}, t_{3}\right)$, que possui relação de intensidade conforme a Equação (9).

$$
I_{3}=\sqrt{I_{1} \times I_{2}}
$$

Substituindo-se o valor da intensidade da Equação (3.8) na Equação (3.9), obteve-se a Equação (10).

$$
\frac{A}{\left(t_{3}+c\right)^{d}}=\sqrt{\frac{A}{\left(t_{1}+c\right)^{d}} \times \frac{A}{\left(t_{2}+c\right)^{d}}}
$$

Isolando a constante " $c$ ", dá-se origem à Equação (11).

$$
c=\frac{t_{3}^{2}-t_{1} \times t_{2}}{t_{1}+t_{2}-2 \times t_{3}}
$$

Para verificação do valor da constante " $\mathrm{c}$ ", foi plotado um novo gráfico, considerando intensidade em função da duração de chuva, porém com a duração deslocada de c, ou seja, $(t+c, l)$. É esperado que os pontos resultantes se aproximem de uma reta.

\subsection{2 - Determinação das constantes " $A$ " e " $d$ "}

No gráfico das intensidades em função das durações deslocadas $(t+c, I)$, os pontos possuem a configuração de uma reta. Essa reta possui coeficiente linear igual a "log(A)" e coeficiente angular igual a "-d". Essa conclusão é obtida ao se aplicar logaritmo dos dois lados da Equação (8), originando a Equação (12).

$$
\log I=\log A-d \times \log (t+c)
$$




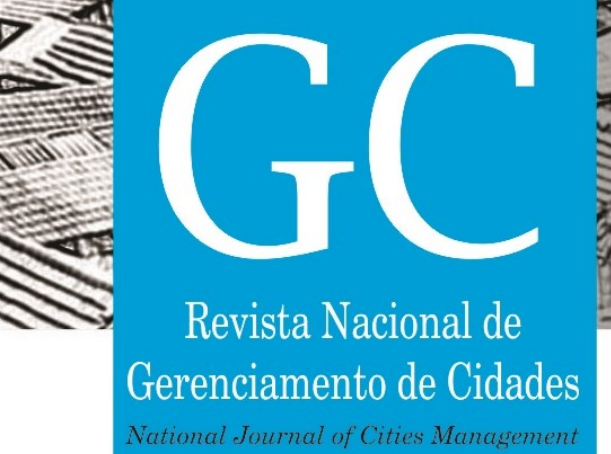

Ao utilizar-se o software Excel, o melhor ajuste aos pontos é um ajuste de potência. Portanto a correlação do ajuste com os coeficientes deve ser realizada a partir da Equação (8) reescrita, o que originou a Equação (13).

$$
I=A *(t+c)^{-d}
$$

\subsection{3 - Determinação das constantes " $a$ " e " $b$ "}

As constantes $a$ e $b$ foram determinadas de modo análogo ao item anterior. Foi aplicado logaritmo nos dois lados da equação (7), gerando a Equação (14).

$$
\log A=\log a+b \times \log \left(T_{R}\right)
$$

Os valores de $\log A$ foram obtidos no item anterior e analogamente, os valores de $a$ e $b$ foram determinados através do método dos mínimos quadrados. Novamente, para ajustes realizados com o software Excel, o melhor ajuste para os pontos é um ajuste de potência que deve ser comparado diretamente com a Equação (7).

\section{6 - Comparação entre as equações I-D-F}

Após a determinação da equação I-D-F com os dados de precipitação atualizados, comparou-se com aquela proposta por Barbassa (1991), representada pela Equação (15).

$$
I=\frac{28,03 * T_{R}^{0,199}}{(t+16)^{0,936}}
$$

Sendo $I$ a intensidade de precipitação ( $\mathrm{mm} /$ minutos); $T_{R}$ o período de retorno (anos); $t$ o tempo de chuva (minutos).

Fixado os períodos de retorno de 1, 5, 10, 20, 50 e 100 anos, calculou-se as intensidades de precipitação a partir das equações atualizada e aquela proposta por Barbassa em 1991, sendo calculadas as diferenças observadas relativas, conforme Equação (16).

$$
D=\frac{I_{A}-I_{\text {Barbassa }}}{I_{\text {Barbassa }}} \times 100
$$

Sendo $D$ a diferença relativa (\%); $I_{A}$ a intensidade de precipitação obtida pela Equação atualizada ( $\mathrm{mm} /$ minuto); $I_{\text {Barbassa }}$ a intensidade de precipitação obtida pela Equação (15) ( $\mathrm{mm} /$ minuto).

\section{4 - RESULTADOS}

\section{1 - Obtenção dos dados pluviométricos}

Os dados referentes à Estação Convencional №83726 do INMET foram obtidos através do endereço eletrônico www.inmet.gov.br. Inicialmente, os dados estavam em formato de texto (.txt) sendo posteriormente convertidos para uma planilha eletrônica do software Excel. Os dados obtidos vão de 01/01/1961 até 31/12/2015, configurando 55 anos de dados disponíveis.

\section{2 - Preparação dos dados pluviométricos}

Os critérios para a eliminação de dados estabelecidos foram: a ocorrência de 10 ou mais dias de falhas de leitura em um mesmo ano e/ou a ocorrência de uma falha no período chuvoso. Trabalhou-se com o fato de o período chuvoso no município ter início no mês de setembro estendendo-se até o mês de março. Os dados levantados mostram que de um total de 54 


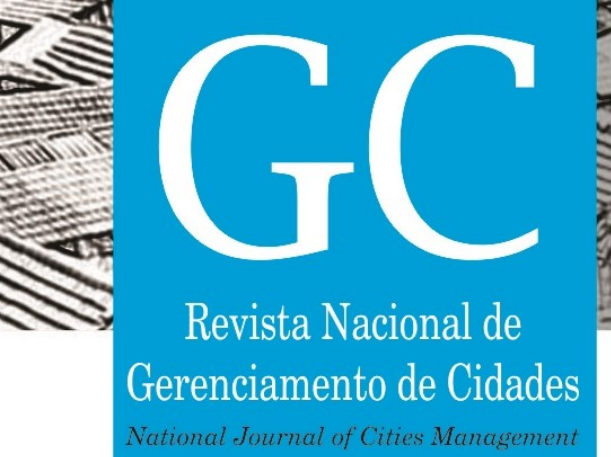

Figura 6: Gráfico bilogaritmo do parâmetro $A$ em função do tempo de retorno $\left(T_{R}\right)$

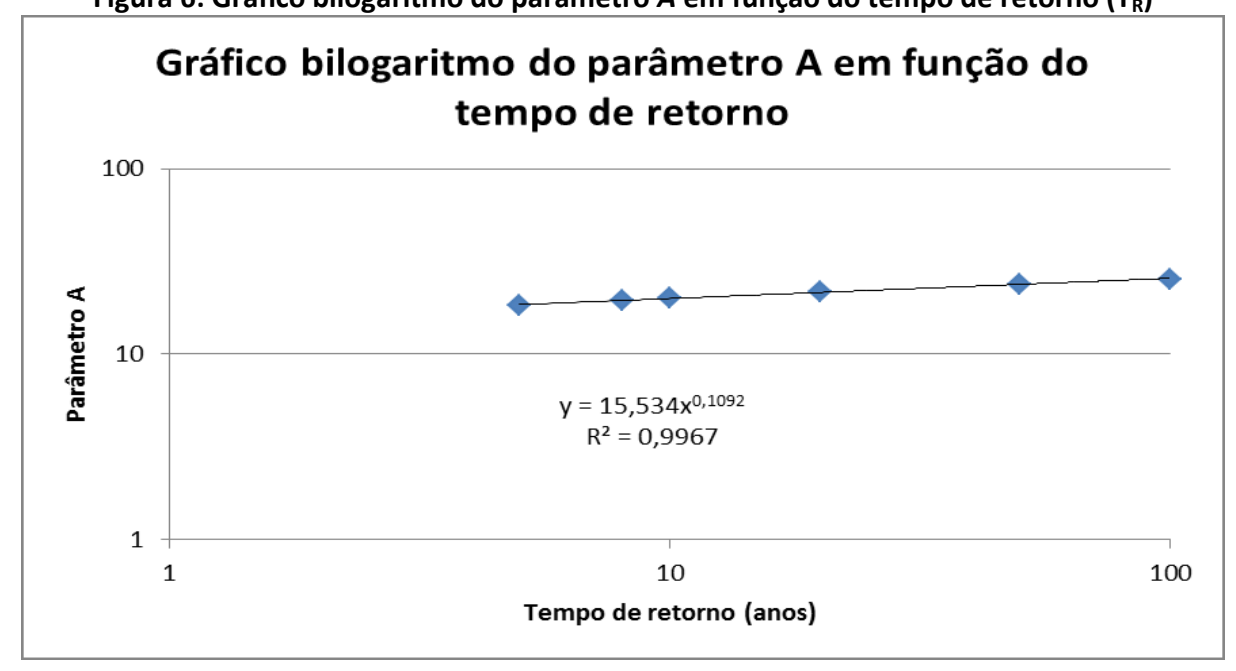

Pela utilização do software Excel, a equação de ajuste dos pontos apresentados na Figura 6 foi comparada diretamente com a Equação (7), obtendo-se os valores das constantes $a$ e $b$, respectivamente, 15,534 e 0,1092 .

\subsection{4 - Determinação da Equação I-D-F}

O formato geral de uma equação I-D-F é apresentado conforme a Equação (3). Substituindo-se as constantes $a, b, c$ e $d$ pelos valores determinados conforme apresentado nas seções anteriores, obteve-se a equação I-D-F atualizada para a cidade de São Carlos (SP), conforme representado pela Equação (17).

$$
I=\frac{15,534 \times T_{R}^{0,1092}}{(t+5)^{0,727}}
$$

Sendo I a intensidade de precipitação ( $\mathrm{mm} /$ minuto); $T_{R}$ o período de retorno (anos); $t$ o tempo de duração da precipitação (minutos).

\subsection{5 - Comparação entre as Equações I-D-F}

As intensidades de chuva obtidas a partir das Equações (15) e (17), foram comparadas, assim como as diferenças relativas foram obtidas a partir da Equação (16), estando os resultados apresentados nos gráficos da Figura 7.

Figura 7: Comparação entre os resultados da equações I-D-F avaliadas para períodos de retorno de: a) 1 ano; b) 5 anos; c) 10 anos; d) 20 anos; e) 50 anos; f) 100 anos 
